\title{
Detection of keratoconus in anterior segment photographed images using corneal curvature features
}

\author{
Marizuana Mat Daud ${ }^{1}$, Wan Mimi Diyana Wan Zaki ${ }^{2}$, Aini Hussain ${ }^{3}$, Haliza Abdul Mutalib ${ }^{4}$ \\ ${ }^{1,2,3}$ Center for Integrated Systems Engineering and Advanced Technologies (INTEGRA), \\ Faculty of Engineering and Built Environment, National University of Malaysia, Malaysia \\ ${ }^{4}$ Optometry and Vision Sciences Programme, School of Healthcare Sciences, Faculty of Health Sciences, \\ National University of Malaysia, Malaysia
}

\begin{tabular}{l}
\hline Article Info \\
\hline Article history: \\
Received Sep 15, 2018 \\
Revised Dec 2, 2018 \\
Accepted Dec 19, 2018
\end{tabular}

Keywords:

Anterior segment

Corneal curvature

Keratoconus

Photographed images

Template disc

\begin{abstract}
Keratoconus is a corneal ectatic disorder with complex aetiology and may induce mild to severe visual impairment and consequently decrease the quality of life. This paper presents a new keratoconus detection method using corneal curvature features to differentiate normal and keratoconus cases. In this study, the eye images known as anterior segmented photographed images (ASPIs) are captured from side view using a smartphone's camera. For the side-view images, the corneal curvature is segmented using spline function to measure the corneal curvature. A template disc method is implemented to quantitatively measure the steepening of the corneal curvature of the captured ASPIs. Parameters obtained from three different template disc methods, namely, nonlinear, $c_{n l}$, crossover point, $c_{c p}$, and trigonometric, $c_{t r}$, are investigated to represent the most suitable curvature feature. SVM is then employed to classify normal and keratoconus eyes. Results reveal that a standalone nonlinear method gives a reliable parameter with $90 \%$ accuracy in classifying the data. However, the classification performance has increased to $99.5 \%$ accuracy with the use of all combined features known as a feature vector, $f_{c}=\left\langle c_{n l}, c_{c p}, c_{t r}\right\rangle$. Additionally, classification with the proposed $f_{c}$ has successfully distinguished normal and keratoconus cases with sensitivity and specificity rates of $99 \%$ and $100 \%$, respectively. The results portray the bright potential of this method in assisting experts during ocular screening specifically to detect keratoconus disease.
\end{abstract}

Copyright $\odot 2019$ Institute of Advanced Engineering and Science. All rights reserved.

\section{Corresponding Author:}

Wan Mimi Diyana Wab Zaki,

Center for Integrated Systems Engineering and Advanced Technologies (INTEGRA),

Faculty of Engineering and Built Environment,

National University of Malaysia, Malaysia.

Email:wmdiyana@ukm.edu.my

\section{INTRODUCTION}

Keratoconus $(\mathrm{KC})$ is an ocular disease involving the progressive and non-inflammatory corneal thinning and steepening of the corneal curvature. However, the non-inflammatory condition has been questioned due to the existence of inflammatory components [1], [2]. The aetiology of $\mathrm{KC}$ is heterogeneous and varies widely depending on geographical factor, family history and races. The prevalence of this disease in the geographical locations of a country with hot climate is higher than that of a country with cooler climate. $\mathrm{KC}$ is also a hereditary disease. However, eye rubbing and allergy are the most consistent findings in contributions to the acceleration of corneal curvature advancement [3]-[6].

$\mathrm{KC}$ is an uncommon disease with 1 case out of 2000 individuals [7]; nonetheless, recent prevalence studies have shown major geographical variations, with the highest cases of 3.59\% reported in Tehran [8] and 
the lowest prevalence of $0.00017 \%$ in Japan [9]. Despite this finding, only a few epidemiological studies have been performed in Asia, especially in Malaysia, because the focus was formerly on other diseases. Only one KC prevalence research has been conducted with a reported prevalence of $1.2 \%$ [10].

Several methods and equipment, such as placido disc, corneal topography and pentacam, can be used to diagnose $\mathrm{KC}$. However, detecting the early phase of $\mathrm{KC}$ where the cornea looks relatively healthy is difficult. The latest extensive equipment includes corneal tomography, corneal biomechanics and in vivo confocal microscopy. These types of equipment, which can detect early signs of $\mathrm{KC}$, provide detailed analysis of the corneal thickness and information on the corneal shape of anterior and posterior segment [11]. However, most of these types of clinical equipment are expensive, heavy and immobile and require experts or well-trained clinicians. In addition to using these types of equipment, ophthalmologists also manually refer to other clinical signs, such as in Rizzuti sign, Vogt striae and Munson [12] sign, as guidelines in diagnosing $\mathrm{KC}$ cases.

With the high pace of technology Internet of Things, researchers can integrate image processing techniques with smartphones via cloud computing. Tareq patented his work on the system and method for ophthalmological imaging using mobile processing device [13]. He developed a device which mimicked a topographical concept using a frustum cone segment, optimisation lens and vertex-angulation positioning light. To the best of our knowledge, only few works reported on KC detection approach using machine learning and image processing methods [13]-[16]. However, these works did not investigate the relationship of corneal curvature of ASPI and the presence of KC.

Numerous studies were conducted on other ocular disease detection methods [17]-[20] using image processing. Anterior segment photographed image (ASPI) is an image taken using a smartphone's camera, a digital camera or any type of cameras. Nayak [21] proposed an automatic classification of normal, cataract and post-cataract of ASPIs using SVM classifier. The algorithm could classify correctly with nearly $90 \%$ accuracy. Raihanah et al. [22] proposed a screening system which can detect and classify pterygium and nonpterygium of ASPIs using image processing technique that was combined with machine learning algorithm. The system performed with accuracy of 95.6\%. Masoud et al. [23] used the template disc method to calculate the tortuosity of retinal blood vessel of fundus images for an automated grading of diabetic retinopathy. They improvised the methods and produced the other two methods, namely, crossover point and trigonometric methods, which are adapted in this work.

A new method of KC detection was developed using the corneal curvature of ASPI. The corneal curvature calculated using template disc is presented in this paper. Qualitatively, corneal curvature measured from a side view of ASPI can be used as an indication of KC severity. The curvature of a side view of a patient's eye image will be measured before validating the eye's condition using a topography machine with an expert's advice. The proposed method is explained in the next section, whereas the results and discussion are discussed in Section 3. Lastly, the conclusion summarises the entire work in Section 4.

\section{PROPOSED METHOD}

The proposed method consists of four parts; preprocessing, segmentation, feature extraction and classification as in Figure 1.

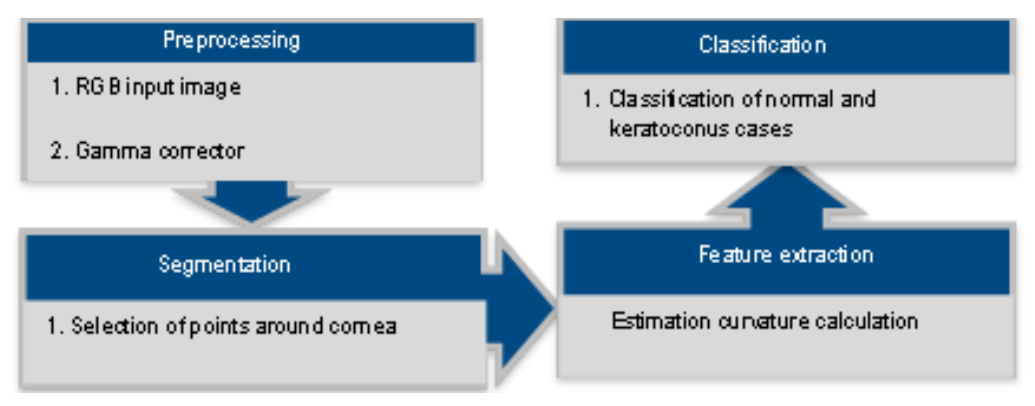

Figure 1. Flow chart of keratoconus detection system

\subsection{Preprocessing}

The ASPIs are captured from the side view of patients' eyes using smartphone cameras. In this study, Huawei P9 and iPhone SE are used to capture the eye images collected from the Ophthalmology Department, Kuala Lumpur Hospital. A total of 106 normal images and $112 \mathrm{KC}$ images are collected, and all 
images are validated by a collaborative optometrist using Corneal Topography (CT). CT generates a different topography map in different eye conditions, such as on Figure 2 (a) and (c) for $\mathrm{KC}$ and normal eye, respectively. Topography map is a colour-based map, where cool colours (cyan to blue) denote flat curves, mild colours (green to orange) specify medium curvature and warm colours (red to black) present high curvature. The range of colour is dependent on the type of equipment. However, we only focus on processing the collected ASPIs using image processing approach.

Corneal is the transparent front structure of the eye. In normal corneas, the light scattering is minimal because of the transparency character; however, for abnormal corneas, the light scattering increases, and consequent loss of corneal transparency occurs [24]. Given this condition, ASPIs have noises such as reflection, uneven illumination and luminosity. Thus, gamma corrector technique is applied to the image during preprocessing to control the luminosity of ASPIs, thereby indirectly enhancing the edges of the cornea and reducing the reflection.

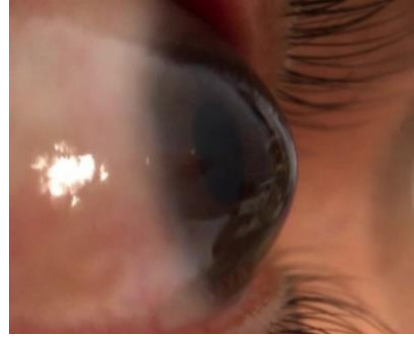

(a)

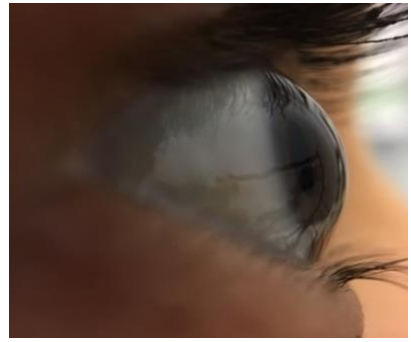

(c)

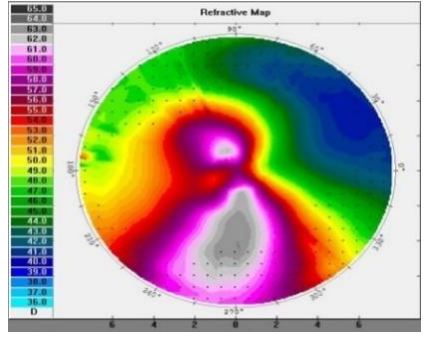

(b)

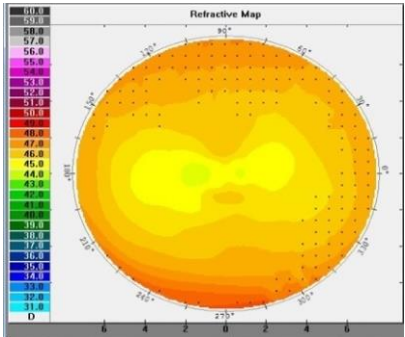

(d)

Figure 2. The side-view image of (a) KC eye with (b) topographical map, and (c) normal eye with (d) topographical map

\subsection{Segmentation}

Image segmentation is a partition of pixels into subregion to simplify the image into something that is remarkable. In this work, a semi-automated segmentation approach is performed by selecting the points $(x, y)$ around the corneal curve, as shown in Figure 3.

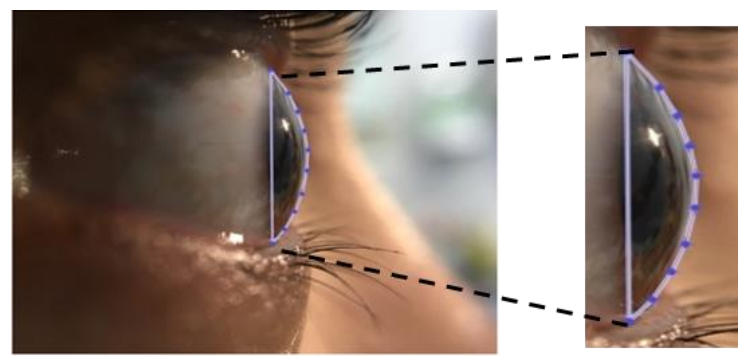

Figure 3. Corneal curvature segmented using Spline function

Spline linear interpolation is then used to connect the points with lines, which are the red line as shown in Figure 4. The selection must be on the edge of the curve with more than five coordinates. 


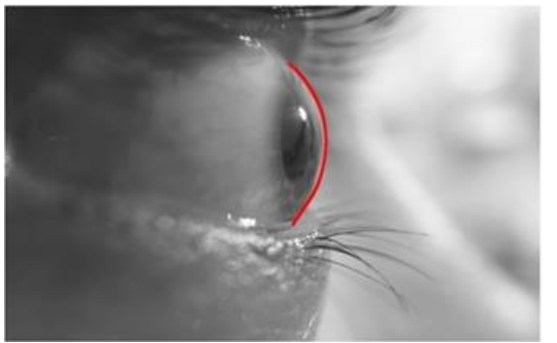

(a) Grayscale Image

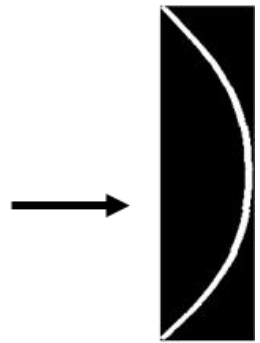

b) Binary image

Figure 4. Corneal curvature extracted into binary image

Our experiment shows that the best number of point selection is 10 points; nevertheless, more points chosen will yield more accurate curvature. Spline is a continuous function which interpolates the data between two points where it is constructed form linear functions interpolating polynomials. The red curve is then extracted to obtain the binary presentation as shown in Figure 4 b. Curvature measurement is calculated in the next section.

\subsection{Feature Extraction - Estimation Curvature Calculation}

Curvature is an indication of local twistedness of a curve [23]-[25]. Several methods exist for measuring curvature of a curve. The curvature is implied as an absolute value of curvature by not considering the rotation of the tangent. The parametric representation when coordinates $x=x(t)$ and $y=y(t)$ are given, [23]

$$
k=\frac{x^{\prime} y^{\prime \prime}+x^{\prime \prime} y^{\prime}}{\left[\left(x^{\prime}\right)^{2}+\left(y^{\prime}\right)^{2}\right]^{3 / 2}}
$$

This approach known as a template disc, where $f(x)$ is a curve, in this case, refers to a corneas curve. A template disc of radius, $d$ is created at the centre of the curve as in Figure 5 to calculate the curvature at a point $(x, y)$.

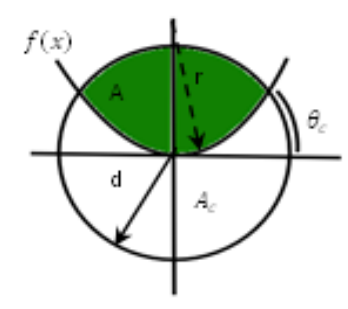

Figure 5. Template disc

The fundamental idea of this method is the relationship between the areas of the curve. The template disc of a suitable radius is placed with its centre at the particular point of the curve. Radius $d$ should be smaller than radius $r$. Mathematically, the position of the template disc and curvature imply the following:

$$
y=\frac{1}{2} c x^{2}+O\left(x^{3}\right)
$$

where $c$ is curvature at origin and $O\left(x^{3}\right)$ denotes higher order term. The normalisation in polar coordinates $(\mathrm{r}, \theta)$ can be written as:

$$
R \sin \theta=\frac{1}{2} C R^{2} \cos \theta+O\left(R^{3} \cos ^{3} \theta\right)
$$

where $R=r / b$ and $C=c / b$. Then, assume that $\theta \approx 0$

$$
\theta(R)=\sin ^{-1}\left[\frac{1}{2} C R+O\left(R^{2}\right)\right]
$$


The angle $\theta$ can now be denoted as the angle between the tangent line passing through the origin while area $A$ can be assumed as the area between the corneal curve and the disc as

$$
a=\int_{0}^{1} R d R \int_{\theta(R)}^{\pi-\theta(R)} d \theta
$$

where $a=A b^{-2}$. Replaced 17164 (4) into (5) while ignoring the high order term and in terms of $c$ yield

$$
c \approx \frac{3 A_{c}}{d^{3}}-\frac{3 \pi}{2 d}
$$

where $A_{k}$ is the complement of $A$. Hence17164 c $\propto A_{k}$ and nonlinear estimation of a curve is defined as

$$
c_{n l} \triangleq A_{c}
$$

The hypothesis of this work is that the more convex of the cornea, the more severe the $\mathrm{KC}$ cornea is. Quantitatively, high $A_{c}$, indicates the high severity of KC. On the basis of [23], Masoud et al. claimed that this method becomes inaccurate when the concern was high estimation curvature. Therefore, through its simplicity, Masoud modified the method using the crossover point, $c_{c p}$ at $\theta_{c}$ and trigonotrimetrical method, $c_{t r}$. The approximations of curvature are as follows

$$
\begin{aligned}
& c_{c p} \triangleq \frac{1}{A^{2}} \\
& c_{t r} \triangleq \frac{2 \sin \theta_{c}}{\cos ^{2} \theta_{c}}
\end{aligned}
$$

We adapt the three features $c_{n l}, c_{c p}$ and $c_{t r}$ into one feature vector, $f_{c}=\left\langle c_{n l}, c_{c p}, c_{t r}\right\rangle$. The feature of $c_{n l}$ and $c_{c p}$ is a form of integration which only rely on area calculation; thus, these features are robust and yield smooth results. The comparison is discussed in the next section.

\subsection{Classification}

Before the evaluation of the proposed methods, one-way analysis of variance (ANOVA) is employed to determine the statistically significant values between normal and $\mathrm{KC}$ corneal curvatures. ANOVA is a statistical method used to find the optimum feature and the existence of significant difference between sets. The results show that only $f_{c 1}=\left\langle c_{n l}\right\rangle$ for the left eye is not significant $(p=0.884)$, as bolded in Table 1, whereas that for right eye is significant $(p<0.05)$ for all features. Thus, all features are fed into Support Vector Machine (SVM) classifier.

The classification of corneal curvature is tested using SVM and decision trees. SVM is a type of pattern classifier based on a novel statistical learning technique [21]. This step is crucial to analyse the curvature either normal or KC. Moreover, this step will evaluate the distinctiveness of the approached algorithm.

Table 1. ANOVA ( $p$-value) Results from Each Feature

\begin{tabular}{ccc}
\hline Features & Right & Left \\
\hline$f_{c 1}<c_{n l}>$ & $1.64 \mathrm{E}-02$ & $\mathbf{8 . 8 4 E - 0 1}$ \\
$f_{c 2}<c_{c p}>$ & $3.60 \mathrm{E}-07$ & $3.40 \mathrm{E}-07$ \\
$f_{c 3}<c_{t r}>$ & $4.80 \mathrm{E}-08$ & $1.30 \mathrm{E}-06$ \\
\hline
\end{tabular}

Three standalone features $f_{c 1}=<c_{n l}>, f_{c 2}=<c_{c p}>, f_{c 3}=<c_{t r}>$ and four combined features vector $\left.f_{c 12}=<c_{n l}, c_{c p}\right\rangle, f_{c 13}=<c_{n l}, c_{t r}>, f_{c 23}=<c_{c p}, c_{t r}>, f_{c}=<c_{n l}, c_{c p}, c_{t r}>$ are used to classify the corneal curvature. Training images are fed into the SVM classifier for training. The trained classifier predicts the testing database without knowing the class of the image. Kernel-based functions include linear kernel function, polynomial kernel function, Gaussian Kernel and Radial Basis Function (RBF). After several testing and experimental on the training data using polynomial kernel function, cubic kernel function is selected in SVM for classification where the input data are transformed into high dimensional space to become discrete compared with the original space.

$$
\mathrm{K}(x, y)=(x \cdot y+1)^{p}
$$


where $p$ is a tunable parameter; for cubic kernel, $p=3$.

A decision tree is an array of "if-then-else" rules for allocation class label to exemplify the data set in the form of tree structure [26]-[27]. A decision node consists of two or more branches, whereas a leaf node represents a decision. The process of developing a tree involves three steps: (a) splitting the data set into subsets, where 4 number of splits are made; (b) pruning is truncating the branches and (c) tree selecting is finding the smallest tree that yields the lowest cross-validated error. The deeper the tree, the fitter the model with more complex of decision rules is. However, the tree must prune the data well; otherwise, it might be over-fitting, which leads into false classification. We use simple decision tree, and the performance results are discussed in the next section.

\section{RESULTS AND DISCUSSION}

The local dataset consisting of 218 images is used to test the reliability of the proposed algorithm using four main steps as explained in Methodology section. The normal and KC ASPIs include the left and right eyes captured from the side view. For these side-view images, the corneal curvature is segmented using spline function to measure the corneal curvature before significant features are extracted. Figures 6 and 7 present the results of 218 images, both eyes, classified using SVM and decision tree. Both classifiers are applied in this work using the standalone and combinations features, $\left\langle c_{n l}, c_{c p}, c_{t r}\right\rangle$ : nonlinear estimation, crossover point and trigonometrical methods. The SVM classifier performs well with the two-combined features $f_{c 12}=\left\langle c_{n l}, c_{c p}\right\rangle$ for both eyes with average accuracy of $98.4 \%$ (Figure 6) compared with standalone feature $f_{c 1}=<c_{n l}>$ and $f_{c 2}=<c_{c p}>$ which is only below than $90 \%$. The weakest combination is $f_{c 23}=\left\langle c_{c p}, c_{t r}>\right.$ for right eye with $72.6 \%$ and for left eye is $65.4 \%$. Overall, the SVM achieves $99.5 \%$ accuracy for both eyes; whereas the decision tree only attains $96.3 \%$ and $83.8 \%$ for right and left eyes, respectively, with the combination of all features $f_{c}=\left\langle c_{n l}, c_{c p}, c_{t r}\right\rangle$. Hence, the corneal curvature method is reliable and competent to classify the $\mathrm{KC}$ and non-KC eye using SVM classifier.

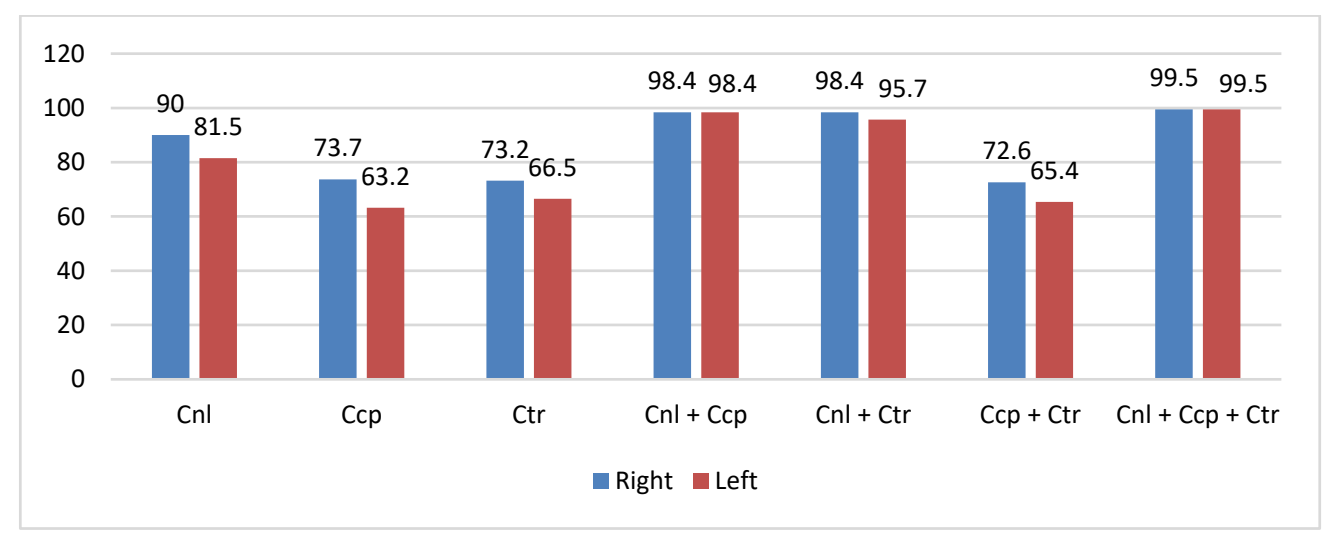

Figure 6. Percentage of accuracy for each and combination features classified using SVM

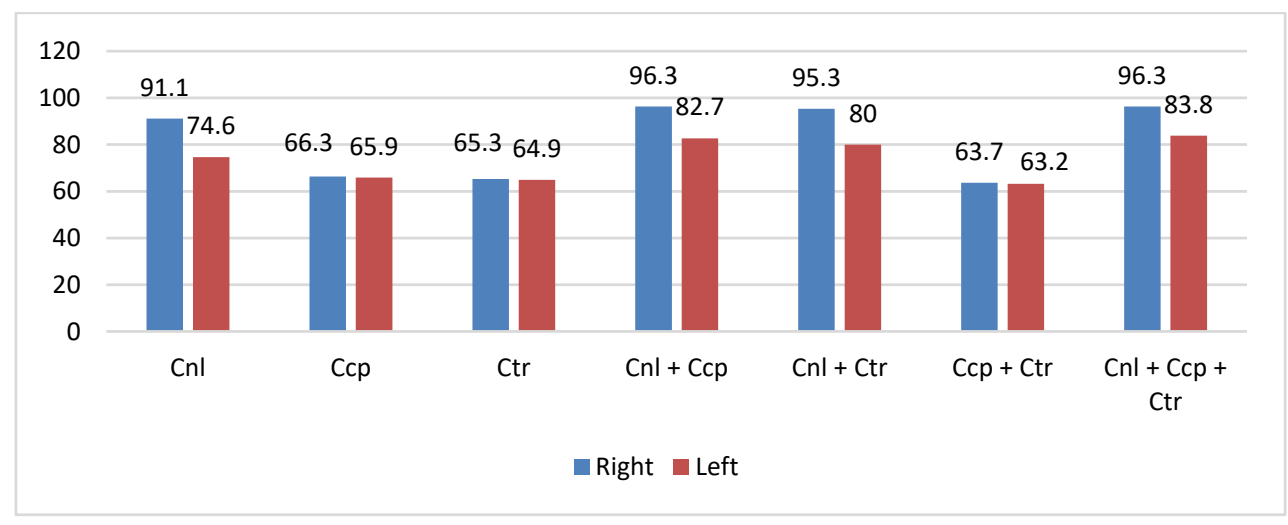

Figure 7. Percentage of accuracy for each and combination features classified using decision tree 
The sensitivity of the approach method is $99 \%$, and the specificity is $100 \%$. In this context, sensitivity indicates that the algorithm can classify the $\mathrm{KC}$ disease in $\mathrm{KC}$ group; whereas the specificity specifies that the system is competent to classify the non-KC disease in normal group. However, only one case of $\mathrm{KC}$ classified in normal group is identified as false negative. The overall performance of this system is reliable in detecting $\mathrm{KC}$ eye.

\section{CONCLUSION}

A new detection method for $\mathrm{KC}$ was proposed using local database and four new modules, namely, preprocessing, feature extraction, segmentation and classification. ASPI was captured from side view of an eye image, and the corneal curvature was calculated using the three parameters of template disc method, namely, nonlinear, crossover point and trigonometrical method. The feature vector, $f_{c}=\left\langle c_{n l}, c_{c p}, c_{t r}>\right.$ was then fed into SVM to classify the eye images with accuracy of $99.5 \%$, sensitivity of $99 \%$ and specificity of $100 \%$. This system may provide a screening platform for $\mathrm{KC}$ detection cases namely people living in rural area, where ophthalmologists are hard to be reached. In line with that purpose, an automated segmentation approach should be developed to produce a user-friendly system in assisting experts during the corneal examination.

\section{ACKNOWLEDGEMENTS}

The authors wish to thank the other members of the Center for Integrated Systems Engineering and Advanced Technologies (INTEGRA) UKM for their support. Funding: This work was supported by the Ministry of Higher Education, Malaysia with grant no. FRGS/1/2016/ICT01/UKM/02/4.

\section{REFERENCES}

[1] Galvis V., et al., "Keratoconus: an inflammatory disorder?” Eye, vol/issue: 29(7), pp. 843-59, 2015.

[2] Gatzioufas Z., et al., "Keratoconus: is it a Non-inflammatory Disease?" Medical Hypothesis, Discovery \& Innovation Ophthalmology Journal, vol/issue: 6(1), pp. 6-7, 2017.

[3] Xu L., et al., "Prevalence and associations of steep cornea/Keratoconus in Greater Beijing. The Beijing Eye Study," PLoS One, vol/issue: 7(7), 2012.

[4] Davidson A. E., et al., "The pathogenesis of keratoconus," Eye (Lond), vol/issue: 28(2), pp. 189-95, 2014.

[5] Sharif R., et al., "Pathogenesis of Keratoconus: The intriguing therapeutic potential of Prolactin-inducible protein," Prog Retin Eye Res., pp. 1-17, 2018.

[6] Carlson A. N., "Keratoconus," Ophthalmology, vol/issue: 116(10), pp. 2036-7, 2009.

[7] Kennedy R. H., et al., "A 48-year clinical and epidemiologic study of keratoconus," Am J Ophthalmol, vol/issue: 101(3), pp. 267-73, 1986.

[8] Hashemi H., et al., "High prevalence and familial aggregation of keratoconus in an Iranian rural population: A population-based study," Ophthalmic Physiol Opt., pp. 1-9, 2018.

[9] U. Tanabe, et al., "Prevalence of keratoconus patients in Japan," Nihon Ganka Gakkai Zasshi, vol/issue: 85(3), pp. 407, 1985.

[10] Mohd Ali B., et al., "Clinical characteristics of keratoconus patients in Malaysia: A review from a cornea specialist centre," J Optom, vol/issue: 5(1), pp. 38-42, 2012.

[11] Gokul A., et al., "Advanced anterior segment imaging in keratoconus: a review," Clin Exp Ophthalmol, vol/issue: 46(2), pp. 122-32, 2018.

[12] Kok Y. O., et al., "Review: keratoconus in Asia," Cornea, vol/issue: 31(5), pp. 581-93, 2012.

[13] Nabhan T. I., "System and method for ophthalmological imaging adapted to a mobile processing device," US 2018/0092534 (Patent), 2018.

[14] Usman M., et al., "Computer Vision Techniques Applied for Diagnostic Analysis of Retinal OCT Images: A Review," Arch Comput Methods Eng., pp. 1-17, 2016.

[15] Toutounchian F., et al., "Detection of Keratoconus and Suspect Keratoconus by Machine Vision," International Multiconference of Engineers and Computer Scientists. Hong Kong, vol. 1, pp. 14-6, 2012.

[16] Arbelaez M. C., et al., "Use of a support vector machine for keratoconus and subclinical keratoconus detection by topographic and tomographic data," Ophthalmology, vol/issue: 119(11), pp. 2231-8, 2012.

[17] Mathew M. K., et al., "Various Cataract Detection Methods-a Survey," Int Res J Eng Technol., vol/issue: 4(1), pp. 1517-9, 2017.

[18] Fuadah Y. N., et al., "Mobile Cataract Detection using Optimal Combination of Statistical Texture Analysis," Int Conf Instrumentation, Commun, Inf Technol Biomed Eng. Bandung, pp. 232-6, 2015.

[19] Li H., et al., "Image based grading of nuclear cataract by SVM regression," SPIE Med Imaging, vol. 6915, pp. 691536-691536-8, 2008.

[20] Patwari A. U., "Detection, Categorization, and Assessment of Eye Cataracts Using Digital Image Processing," The First International Conference on Interdisciplinary Research and Development. Thailand, pp. 1-5, 2011. 
[21] Nayak J., "Automated classification of normal, cataract and post cataract optical eye images using SVM classifier," Proc World Congr Eng., vol. 1, pp. 23-5, 2013.

[22] Wan Zaki W. M. D., et al., "Automated pterygium detection method of anterior segment photographed images," Comput Methods Programs Biomed, vol. 154, pp. 71-8, 2018.

[23] Aghamohamadian S. M., et al., "A Novel Curvature Based Algorithm for Automatic Grading of Retinal Blood Vessel Tortuosity," IEEE J Biomed Heal Informatics, vol/issue: 20(2), pp. 586-595, 2015.

[24] Meek K. M. and Knupp C., "Corneal structure and transparency," Prog Retin Eye Res., vol. 49, pp. 1-16, 2015.

[25] T. F. Banchoff and S. T. Lovett, "Differential geometry of curves and surfaces," First Edition, New York: A K Peters/CRC Press, 2010.

[26] Twa M. D., et al., "Automated decision tree classification of corneal shape," Optometry and vision science: official publication of the American Academy of Optometry, vol/issue: 82(12), pp. 1038, 2005.

[27] Twa M. D., et al., "Decision tree classification of spatial data patterns from videokeratography using Zernike polynomials," Proceedings International Conference on Data Mining. San Francisco, pp. 3-12, 2003.

\section{BIOGRAPHIES OF AUTHORS}

\begin{tabular}{|c|c|}
\hline & $\begin{array}{l}\text { Marizuana Mat Daud is a PhD candidate at Universiti Kebangsaan Malaysia. Her research } \\
\text { interests include image processing with machine learning focusing on biomedical field. More } \\
\text { specifically, her work examines how image processing with machine learning techniques could } \\
\text { contribute towards better and more efficient ways of analyzing and understanding medical } \\
\text { images. Apart from that, her research interest also includes assistive technologies for people with } \\
\text { disabilities. }\end{array}$ \\
\hline & $\begin{array}{l}\text { Wan Mimi Diyana Wan Zaki obtained her Bachelor degree (Electronics Engineering) in 2000, } \\
\text { Master degree (Engr. Sc.) in } 2005 \text { and PhD degree in 2012, all from the Multimedia University } \\
\text { (MMU), Cyberjaya, Malaysia. She is currently a researcher and senior lecturer at the Centre of } \\
\text { Integrated Systems Engineering and Advanced Technology (INTEGRA), Universiti Kebangsaan } \\
\text { Malaysia (UKM), which she joined in } 2008 \text {. Her research specialisation is in biomedical } \\
\text { engineering, and her research interests include intelligent systems, image processing and IoT } \\
\text { related healthcare technology. }\end{array}$ \\
\hline & $\begin{array}{l}\text { Aini Hussain obtained her B.Sc. in Electrical Engineering from Louisiana State University } \\
\text { (LSU), Baton Rouge, USA; M.Sc. in Systems and Control from the University of Manchester } \\
\text { Institute of Science and Technology (UMIST), Manchester, U.K., and Ph.D. in Electrical and } \\
\text { Electronic Engineering from the National University of Malaysia in 1985, } 1991 \text { and 1997, } \\
\text { respectively. She is a Professor and currently, the Chair of the INTEGRA research center also } \\
\text { known as "Centre for Integrated Systems Engineering and Advanced Technologies". } \\
\text { Her research, for which she has received funding, focuses on Intelligent Systems and Image } \\
\text { Processing. Her current research interests are in machine learning, pattern recognition and video } \\
\text { \& image processing. }\end{array}$ \\
\hline & $\begin{array}{l}\text { Haliza Abdul Mutalib is an Associate Professor in Centre for Community Health, Faculty of } \\
\text { Health Sciences, National University of Malaysia since year 2008. She specializes in Contact } \\
\text { Lenses \& Corneal Morphology. Her current research interests are on morphological changes in } \\
\text { contact lens wear, observation of physiological corneal cell changes using confocal microscopy, } \\
\text { therapeutic contact lenses, contact lens and cleaning regime effect to ocular status and other } \\
\text { topics related to contact lenses. She was conferred MSc in Ophthalmology \& Vision Sciences } \\
\text { (1997) and a PhD (Optometry) from UMIST, United Kingdom in 2000. She managed to } \\
\text { complete her PhD in } 23 \text { months and was awarded with VDC Peter Abel Award (Germany) for } \\
\text { Best Thesis in Contact Lens Research. }\end{array}$ \\
\hline
\end{tabular}

